\title{
Medicare Certified Long Term Care Hospital
}

National Cancer Institute

\section{Source}

National Cancer Institute. Medicare Certified Long Term Care Hospital. NCI Thesaurus. Code C99984.

A medicare-certified medical facility equipped and staffed to provide prolonged care to patients, usually in cases when rehabilitation or total patient care are necessary. (ACC) 